\title{
Mixed Function Oxidase
}

National Cancer Institute

\section{Source}

National Cancer Institute. Mixed Function Oxidase. NCI Thesaurus. Code C16865.

These enzymes use molecular oxygen catalyze the donation of one oxygen atom to a substrate specific site, while the other atom is donated to the generation of water. The enzyme uses molecular oxygen as the oxygen source instead of water, and is critical for several endocrine function operations. 